\title{
Early-onset preeclampsia - the impact of antiphospholipid antibodies on disease severity: a case-control study
}

\author{
Roni Zemet ${ }^{1}$, Micha Baum², Hadas Ofer Friedman ${ }^{3}$, Iris Morag ${ }^{1}$, Mordechai Dulitzki ${ }^{1}$, and \\ Michal Simchen ${ }^{1}$ \\ ${ }^{1}$ Sheba Medical Center at Tel Hashomer \\ ${ }^{2}$ Sheba Medical Center \\ ${ }^{3}$ Beilinson medical center
}

September 10, 2020

\begin{abstract}
Objective: To evaluate whether early-onset severe preeclampsia prior to 34 weeks' gestation is clinically different when associated with antiphospholipid antibodies. Design: A retrospective case-control study. Setting: Single university-affiliated tertiary referral center Population: 55 women with singleton pregnancies who delivered prior to 34 weeks' gestation due to preeclampsia with severe features. Methods: Out of the 101 women with preeclampsia with severe features, the antiphospholipid antibodies status of 55 was available for analysis. The study group comprised 20 women with positive antiphospholipid antibodies (positive-aPL group), while the control group comprised 35 women without antiphospholipid antibodies (negative-aPL group). Main outcome measures: Obstetric and neonatal outcomes, laboratory results and pregnancy complications. Results: Positive-aPL women were hospitalized earlier (29, IQR 26.3-32, vs. 32, IQR 28-33 weeks gestation, $\mathrm{p}=0.05$ ), gave birth at a significantly earlier gestational age (30, IQR 28.3-32.8 vs. 33, IQR 30-34, $\mathrm{p}=0.02)$ with a lower mean birth weight (1266.7 \pm 579.6 vs. $1567.3 \pm 539.7$ grams, $\mathrm{p}=0.058$ ) compared with negative-aPL women. Furthermore, platelet nadir was significantly lower for positive-aPL compared with negative-aPL women $(97.2 \pm 49.7103 / \mu \mathrm{L}$ vs $141.3 \pm 61.13 / \mu \mathrm{L}, \mathrm{p}<0.001)$ and maximal serum creatinine was higher $(1.02 \pm 0.32 \mathrm{mg} / \mathrm{dL}$ vs. $0.92 \pm 0.13 \mathrm{mg} / \mathrm{dL}, \mathrm{p}=0.03)$. Rates of neonatal complications were low and comparable between groups, although there was a trend for higher perinatal mortality among study group infants. Conclusions: The presence of antiphospholipid antibodies in women with early-onset preeclampsia with severe features is associated with earlier, more severe multi-organ involvement. Expedited screening for antiphospholipid antibodies in cases of early-onset severe preeclampsia may be considered.
\end{abstract}

\section{Tweetable abstract}

The presence of aPL antibodies in women with early-onset preeclampsia is associated with more severe multi-organ involvement.

Key words: Antiphospholipid antibodies; antiphospholipid syndrome; early-onset preeclampsia; HELLP syndrome; preeclampsia; pregnancy complications; obstetrics outcomes; neonatal outcomes; thrombophilia.

\section{Introduction}

Hypertensive disorders of pregnancy remain one of the leading causes of maternal and perinatal morbidity and mortality. It is estimated that preeclampsia complicates $2-8 \%$ of pregnancies, ${ }^{1-4}$ and is classically characterized by new onset of hypertension and proteinuria ${ }^{1-3,5}$ although clinical presentations may vary. ${ }^{6}$ Preeclampsia can be defined as either non-severe or severe, depending on the severity of hypertension or the presence of specific clinical or laboratory criteria (i.e. HELLP syndrome, eclampsia, etc). ${ }^{7-10}$ Preeclampsia with severe features may lead to end organ damage, including the central nervous system, cardiovascular 
system, coagulation, liver and kidney. ${ }^{11}$ Early-onset preeclampsia before 34 weeks' gestation is generally associated with a more severe clinical course for the mother, as well as neonatal complications related to prematurity. ${ }^{12-13}$ Although the exact pathogenesis of preeclampsia in not fully appreciated, several clinical risk factors have been identified, such as nulliparity, previous history of preeclampsia, multifetal gestation, pregestational diabetes, chronic hypertension, renal disease, obesity, thrombophilia, systemic lupus erythematosus as well as antiphospholipid antibody syndrome. ${ }^{14-16}$

Antiphospholipid syndrome (APS) is an autoimmune, hypercoagulable state caused by the presence of antiphospholipid antibodies (aPL antibodies). aPL antibodies have been associated with a variety of obstetric complications, including recurrent pregnancy loss, and placental mediated complications, such as early-onset preeclampsia, intrauterine growth restriction, placental insufficiency, placental abruption, preterm delivery and late fetal loss. ${ }^{17-23}$

Despite the amassed body of evidence describing the association between aPL antibodies and preeclampsia, the significance of the presence of aPL antibodies in preeclamptic women with severe features has not been thoroughly elucidated. Specifically, it is unclear whether early-onset severe preeclampsia before 34 weeks gestation presents with different clinical features when associated with antiphospholipid antibodies.

Therefore, the aim of our study was to determine if the presence of aPL antibodies is associated with different maternal or neonatal clinical characteristics in those with early-onset preeclampsia who delivered prior to 34 weeks.

\section{Materials and Methods}

\section{Study design and population}

This is a retrospective case-control study comparing the clinical characteristics and outcomes of singleton pregnancies in women with preeclampsia with severe features who were delivered prior to 34 weeks of gestation with and without aPL antibodies. The study was conducted in a single university-affiliated tertiary referral center. Data were collected from the medical chart of pregnancies presenting with the above diagnosis between 2005 and 2014.

All women who gave birth to a singleton neonate at or before 34 weeks gestation with the discharge diagnosis of severe preeclampsia were considered for inclusion in the study. The primary exposure of interest consisted of the presence or absence of aPL antibodies. Medical records of eligible study participants were scanned to document the results of aPL antibodies work-up. Women with no evidence of aPL antibodies work-up results in their medical charts were contacted by phone after delivery and invited to complete an aPL antibodies work-up in our institution. Therefore, the presence or absence of aPL antibodies was documented after delivery and did not impact on clinical management during pregnancy or delivery.

Inclusion criteria included: 1 . Delivery at or before 34 completed weeks of gestation because of preeclampsia with severe features; 2 . Singleton pregnancy.

Exclusion criteria included: 1. Multifetal pregnancy; 2. Lack of available data on pregnancy and delivery; 3. Pregnancies complicated with congenital anomalies; 4. Lack of information on the presence or absence of aPL antibodies.

Presence of aPL antibodies was defined as any single positive antiphospholipid test, including lupus anticoagulant (LAC), anticardiolipin (aCL) IgG or IgM, and anti- $\beta 2$-glycoprotein1 IgG or IgM. The presence of LAC was confirmed by two different clotting assay principles: activated partial thromboplastin time (APTT)-based assays and dilute Russell's viper venom time (dRVVT). The presence of aCL and anti- $\beta 2 \mathrm{GP} 1$ antibodies of IgG or IgM isotype was tested by standardized enzyme-linked immunosorbent assay (ELISA). Moderate to high levels of aCL IgM/IgG and anti- $\beta 2 \mathrm{GP} 1 \mathrm{IgG} / \mathrm{IgM}$ were defined as $>99^{\text {th }}$ percentile or $>40$ GPL or MPL units, according to the cutoff levels recommended in the revised Sapporo criteria. ${ }^{20}$ 
Within this cohort of pregnant women with preeclampsia with severe features who gave birth prior to 34 completed weeks, we therefore compared two groups: the study group - aPL-positive group - comprised of women with evidence for the presence of aPL antibodies, while the control group - aPL-negative group included women with no evidence of aPL antibodies. Confirmatory repeat testing of aPL antibodies was recommended to all participants, but the results were not available for this study.

\section{Outcome measures}

Demographic and clinical data were collected from the electronic records of all patients and neonates. The following characteristics were determined: Maternal age, gravidity, parity, maternal disease (i.e. diabetes mellitus, chronic hypertension), length of pre- and post-delivery hospital stay; blood pressure measurements; blood work results; symptoms; pregnancy complications (i.e. intra-uterine growth restriction, placental abruption); mode of delivery; gestational age at delivery; birth weight; Apgar score; sex. Also recorded were the following neonatal complications: respiratory distress syndrome (RDS), bronchopulmonary dysplasia (BPD), intraventricular hemorrhage (IVH), periventricular leukomalacia (PVL), retinopathy of prematurity (ROP) and necrotizing enterocolitis (NEC) as well as neonatal death.

\section{Clinical definitions}

Preeclampsia with severe features was defined according to the ACOG criteria. ${ }^{1}$ Women were diagnosed with preeclampsia if they had new-onset elevated blood pressure (with systolic blood pressure greater than $140 \mathrm{mmHg}$ and/or diastolic blood pressure greater than $90 \mathrm{mmHg}$ on two occasions at least 4 hours apart) at or after 20 weeks of gestation or acute worsening of chronic blood pressure, with or without proteinuria (urinary excretion of 300mg protein/day). Severe features included systolic blood pressure greater than $160 \mathrm{mmHg}$ and/or diastolic blood pressure greater than $110 \mathrm{mmHg}$, platelet count $<100,000 / \mathrm{ml}$, liver enzymes twice the normal concentration or symptoms of liver failure, right upper quadrant or epigastric pain, serum creatinine $>1.1 \mathrm{mg} / \mathrm{dl}$, pulmonary edema, and/or new-onset cerebral or visual disturbances.

HELLP syndrome was defined by the clinical presentation of hemolysis, elevated liver enzymes, and low platelet count. We used the following criteria to make a diagnosis: lactate dehydrogenase (LDH) elevated to $600 \mathrm{IU} / \mathrm{L}$ or more, aspartate aminotransferase (AST) and alanine aminotransferase (ALT) elevated more than twice the upper limit of normal, and the platelets count less than $100,000 \times 10^{9} / \mathrm{L}^{24}$

Birth weight was obtained immediately after birth using a standard electrical scale. Diagnosis of small for gestational age (SGA) was made using recorded birth weight and customized local nomograms ${ }^{25}$ and defined as birth weight less than the 10th percentile for gestational age and sex. Respiratory distress syndrome (RDS) was defined as the presence of clinical signs of respiratory distress (tachypnea, retractions, flaring, grunting, or cyanosis), with a requirement for supplemental oxygen with a fraction of inspired oxygen of more than 0.21 and a chest radiograph showing hypo-aeration and reticulogranular infiltrates. Respiratory support was characterized as continuous positive airway pressure (CPAP) or mechanical ventilation oxygen supplementation. Jaundice was defined as hyperbilirubinemia requiring treatment, and sepsis was confirmed by positive blood cultures. Hypoglycemia was defined as a glucose level of less than $40 \mathrm{mg}$ per deciliter at any time.

\section{Statistical analysis}

Continuous variables are presented as means and standard deviation or as median and inter-quartile range (IQR). Normality of the data was tested using the Shapiro-Wilk or Kolmogorov-Smirnov tests. Univariate comparison of continuous variables with normal distribution and dichotomous data or other continuous variables were performed with the use of student's t test and Pearson correlation, respectively. Comparison between continuous variables not normally distributed was conducted with Mann-Whitney U test and Spearman's rank correlation. Categorical variables are presented as numbers and percentages. The Pearson's chi-square and Fisher's exact tests were used for comparison of categorical variables. The significance threshold was set as $\mathrm{p}<0.05$. Statistical analyses were conducted using the IBM Statistical Package for the Social Sciences (IBM SPSS for windows v.20; IBM Corporation Inc, Armonk, NY, USA). 
The study protocol was approved by the Institutional Review Board at the Sheba Medical Center (9683-12$\mathrm{SMC})$, and written informed consent was obtained from all participants.

\section{Results}

Out of 101 women with singleton pregnancies giving birth prior to 34 completed weeks of gestation due to preeclampsia with severe features during the study period, the aPL antibodies status of 55 women was available for analysis. The median gestational age at diagnosis was 31 (IQR 28-33), and the median gestational age at delivery was 32 (IQR (29-34) with a mean birth weight of $1458 \pm 568$ grams. The study group - aPL-positive group - comprised of 20 women, while the aPL-negative group comprised of 35 women who served as controls. The antibodies distribution of the aPL-positive group is presented in Table 1.

Table 2 displays the demographic and clinical characteristics of the study and control groups. All participants were Caucasian. Women in the study group were younger than controls $(30.15 \pm 4.64$ vs. $34.34 \pm 7.02, \mathrm{p}=0.01)$, but with higher gravidity (3, IQR 1.25-4.75, in the study group vs. 2, IQR 1-2, in controls, p=0.01). Medical history of chronic hypertension and diabetic mellitus, pre-gestational or gestational, were comparable between the two groups. There were 2 cases of systemic lupus erythematosus among study group cases and one among controls. Additionally, there was one case of glomerulonephritis and one case of Behçet's disease among the study group.

The obstetric outcomes and pregnancy complications for pregnancies in aPL-positive and aPL-negative women are presented in Table 3. Median gestational age at hospitalization was three weeks earlier in aPLpositive women compared with aPL-negative women (29, IQR 26.28-32, vs. 32, IQR 28-33 weeks gestation, respectively, $\mathrm{p}=0.05)$. aPL-positive women delivered at significantly earlier gestational age (30, IQR 28.3-32.8 weeks, vs. 33, IQR 30-34 weeks, $\mathrm{p}=0.02$ ) with lower mean neonatal birth weight compared with those with negative aPL antibodies (1266.7 \pm 579.6 grams vs. $1567.3 \pm 539.7$ grams, respectively, $\mathrm{p}=0.058)$. There were two peripartum fetal deaths in our cohort, both to aPL-positive mothers. One case was an antepartum fetal death at 27 weeks gestation of a severely growth-restricted fetus, and the other case was a severely growthrestricted neonate born at 24 weeks gestation who died at two days of age from complications of prematurity. The rate of cesarean section was high and comparable between the groups. Furthermore, women in the aPLpositive group were hospitalized for a longer period after delivery compared with aPL-negative group women (9, IQR 6-12, vs. 7, IQR 6-9 days, respectively, $\mathrm{p}=0.03$ ).

In order to assess the severity of the disease, we evaluated clinical and laboratory characteristics of preeclampsia with severe features in both groups. Although maximal systolic blood pressure was lower for aPL-positive women compared with aPL-negative controls $(149.72 \pm 22.6$, vs. $170.03 \pm 23.1, \mathrm{p}=0.004)$, overall disease severity was more significant for aPL-positive women. Platelet nadir was significantly lower for aPL-positive compared with aPL-negative women (mean $97.2 \pm 49.710^{3} / \mu \mathrm{L}$ vs. $141.3 \pm 61.1^{3} / \mu \mathrm{L}$, respectively, $\mathrm{p}<0.001$ ) and maximal serum creatinine was higher $(1.02 \pm 0.32 \mathrm{mg} / \mathrm{dL}$ vs. $0.92 \pm 0.13 \mathrm{mg} / \mathrm{dL}, \mathrm{p}=0.03)$. Moreover, both cases of eclampsia occurred in the aPL-positive women. Table 3 outlines the frequency of severe pregnancy complications in both groups.

Twenty neonates were born to aPL-positive mothers, and 35 to aPL-negative mothers (Table 4). Rates of complications of prematurity were comparable between groups, except for higher rates of retinopathy of prematurity in women with positive aPL antibodies $(30.0 \%$ vs. $5.7 \%, \mathrm{p}=0.02)$.

As mentioned above, there were two antepartum fetal deaths in our cohort, both to study group mothers. Neonatal death before discharge occurred in one neonate born to an aPL-positive mother and one of 35 neonates born to aPL-negative mothers. Therefore, overall perinatal mortality was higher for aPL-positive women compared with aPL-negative women, although not statistically significant (3 out of 20 (15\%) vs. 1 out of $35(2.8 \%), \mathrm{p}=0.13)$.

Discussion 


\section{Main Findings}

The results of our study indicate that when early-onset preeclampsia prior to 34 weeks gestation occurred in aPL-positive women it was clinically more severe than in aPL-negative women. Women with positive aPL antibodies presented and were hospitalized earlier, delivered at a significantly earlier gestational age and with lower mean birth weight compared with women with negative aPL antibodies. Furthermore, platelet nadir was significantly lower for aPL-positive women and maximal serum creatinine was higher, two characteristics of preeclampsia with severe features. Although the study lacked the power to detect statistical differences in rare events, the rate of fetal/neonatal death and eclampsia was higher in women with positive aPL antibodies.

\section{Interpretation}

Preeclampsia is one of the leading causes of maternal morbidity and mortality, and contributes significantly to neonatal morbidity and mortality, mainly due to indicated preterm delivery. ${ }^{26}$ Preeclampsia constitutes one of the pregnancy complications accompanying APS, and 11-17\% of women with preeclampsia will test positive for aPL antibodies. ${ }^{17,27-30}$ In addition to an increased risk for vascular thrombosis in women with APS, the association between aPL antibodies and placenta mediated pregnancy complications, including early-onset preeclampsia, is part of the definition of APS. ${ }^{17-23,31}$ Nevertheless, the severity of preeclampsia with regard to aPL antibodies status has not been elucidated. The novelty of the present study is in the association of these antibodies with the severity of early onset preeclampsia, including obstetric and neonatal outcomes.

The association between aPL antibodies and preeclampsia is well established, both from cohort and casecontrol studies, ${ }^{32}$ as well as two large meta-analyses. The meta-analysis by do Prado et al. included 12 studies, and found a pooled odds ratio (OR) for association of aCL with preeclampsia of 2.86 (95\% CI 1.37-5.98). Pooled OR for aCL with preeclampsia with severe features was 11.15 (95\% CI 2.66-46.75). In studies involving patients with mild preeclampsia, this association was weaker, indicating a dose-response relationship and supporting the possibility of a causal association..$^{33} \mathrm{~A}$ second meta-analysis by Abou-Nassar et al. included 28 studies, and found an association between LAC and preeclampsia in case-control studies (OR 2.34; 95\% CI 1.18-4.64), but not in cohort studies. aCL IgG was associated with preeclampsia in casecontrol studies (OR 1.52, 95\% CI 1.05-2.2), and anti- 32 GP1 was associated with preeclampsia in cohort studies (OR 19.14, 95\% CI 6.34-57.77). ${ }^{34}$ These associations suggest a correlation between preeclampsia and antiphospholipid antibodies. In addition, a prospective evaluation of 1155 women revealed that women with antiphospholipid antibodies had an increased risk for pregnancy-induced hypertension (OR 5.5; 95\% CI 2.3-13.5) and severe pregnancy-induced hypertension (OR 8.1 for any aPL antibody, 95\% CI 2.2-29.4; and OR 143 for multi-positive). ${ }^{20}$ These epidemiologic findings are consistent with animal studies. ${ }^{32}$ Murine studies on the effects of aPL antibodies have found complement mediated fetal injury in APS, ${ }^{35-38}$ while placentas from human pregnancies affected by APS also show increased complement deposition. ${ }^{35,39}$ Hence, complement activation is a possible pathway through which aPL antibodies may lead to adverse pregnancy outcomes.

According to the American College of Obstetrics and Gynecology, although preterm preeclampsia with severe features and early-onset placental insufficiency are indicated as clinical criteria for the diagnosis of APS by expert consensus, ${ }^{31}$ insufficient evidence exists to support screening for the presence of aPL antibodies and treatment of women with these conditions to improve subsequent pregnancy outcomes. ${ }^{17}$ The findings in our study suggest that the presence of aPL antibodies in women with preeclampsia with severe features that deliver prior to 34 weeks presents at an earlier gestational age and affects the severity of the disease. These findings might strengthen the recommendation to screen for aPL antibodies in cases of past or present early-onset preeclampsia with severe features. Furthermore, the presence of aPL antibodies should be cause for diligent scrutiny of these pregnancies to detect complications and evidence for end-organ damage in a timely fashion, thus allowing for adequate intervention for mother and fetus.

\section{Strengths and Limitations}

Several limitations of our study should be acknowledged. First, the study is limited by the relatively small 
number of participants in the study. Second, the study is retrospective in design, therefore susceptible to confounding factors. The retrospective observational design of the study precludes comments on causality in the association between aPL antibodies and the severity of preeclampsia and preterm birth. Third, the study lacks adequate power to detect differences in rare events, i.e. neonatal mortality and significant neonatal morbidity. Nevertheless, since women with positive-aPL and early-onset preeclampsia with severe features delivered significantly earlier compared with those with negative-aPL, it is possible that a larger sample size would have found a statistically significant benefit in neonatal outcome. Lastly, aPL antibodies were determined once due to lack of success at obtaining repeat testing. It is clinically important to pursue repeat confirmatory testing, but we acknowledge the difficulties in obtaining it. Of note, Erkan et al. showed that positive aPL antibodies remained stable in $75 \%$ of cases. ${ }^{40}$ We promote caution in defining aPL-positive women as suffering from full-blown APS. Nevertheless, it is interesting to note that although not complying with the definition of APS, these women still demonstrate important clinical differences when compared with aPL-negative women.

Among the strengths of our study is the novel association between the presence of aPL antibodies and the clinical severity of early-onset preeclampsia. We specifically targeted a pre-defined phenotype of preeclampsia that resulted in preterm delivery prior to 34 weeks gestation to delineate severe disease. All aPL antibodies were tested using standardized techniques, and pregnancy outcomes were diagnosed using guidelines and international criteria or definitions.

\section{Conclusion}

In conclusion, our findings confirm that the presence of antiphospholipid antibodies in women with earlyonset preeclampsia with severe features is associated with earlier, more severe multi-organ involvement. Early screening for aPL antibodies in cases of preeclampsia should be encouraged, and optimal combination therapy, in the current or the following pregnancy, should be considered in women with positive aPL antibodies to improve pregnancy outcomes. To generalize our conclusions and search for causality in the association between aPL antibodies and the severity of preeclampsia, high quality prospective multicenter randomized control study should be conducted.

Acknowledgments: None.

Disclosure of Interests: The authors report no conflict of interest.

Ethics approval: The study protocol was approved by the Institutional Review Board at the Sheba Medical Center (9683-12-SMC) on June 09, 2015, and written informed consent was obtained from all participants.

Funding: None

\section{Contribution to Authorship}

RZ - Substantial contribution to acquisition, analysis and interpretation of data

Drafting the article submitted and revising it for important intellectual content

Approval of the final version to be published

MB - Drafting the article submitted and revising it for important intellectual content

Approval of the final version to be published

HOF - Substantial contribution to acquisition, analysis and interpretation of data; Approval of the final version to be published

IM - Substantial contribution to conception and design of the project

Substantial contribution to acquisition, analysis and interpretation of data

Approval of the final version to be published 
MD - Substantial contribution to conception and design of the project

Substantial contribution to acquisition, analysis and interpretation of data

Drafting the article submitted and revising it for important intellectual content

Approval of the final version to be published

MJS - Substantial contribution to conception and design of the project

Substantial contribution to acquisition, analysis and interpretation of data

Drafting the article submitted and revising it for important intellectual content

Approval of the final version to be published

References

1. ACOG Practice Bulletin No. 202: Gestational Hypertension and Preeclampsia. Obstet Gynecol. 2019 Jan; 133(1): e1-e25.

2. Steegers EA, von Dadelszen P, Duvekot JJ, Pijnenborg R. Pre-eclampsia. Lancet 2010; 376: 631-44

3. Ghulmiyyah L, Sibai B. Maternal mortality from preeclampsia/eclampsia. Semin Perinatol 2012; 36: $56-9$.

4. Lindheimer MD, Taler SJ, Cunningham FG. Hypertension in pregnancy. J Am Soc Hypertens. 2008 Nov-Dec; 2(6): 484-94

5. Uzan J, Carbonnel M, Piconne O, Asmar R, Ayoubi JM. Preeclampsia: pathophysiology, diagnosis, and management. Vasc Health Risk Manag. 2011; 7: 467-74.

6. Homer CS, Brown MA, Mangos G, Davis GK. Non-proteinuric preeclampsia: a novel risk indicator in women with gestational hypertension. J Hypertens 2008; 26: 295-302

7. Barton JR, Sibai BM. Diagnosis and management of hemolysis, elevated liver enzymes, and low platelets syndrome. Clin Perinatol 2004; 31: 807-33, vii.

8. Sibai BM, Ramadan MK, Usta I, Salama M, Mercer BM, Friedman SA. Maternal morbidity and mortality in 442 pregnancies with hemolysis, elevated liver enzymes, and low platelets (HELLP syndrome). Am J Obstet Gynecol. 1993 Oct;169(4):1000-6.

9. Sibai BM. Diagnosis, controversies, and management of the syndrome of hemolysis, elevated liver enzymes, and low platelet count. Obstet Gynecol. 2004 May;103(5 Pt 1):981-91.

10. Sibai BM. Diagnosis, prevention, and management of eclampsia. Obstet Gynecol. 2005 Feb; 105(2): 402-10

11. von Dadelszen P, Payne B, Li J, Ansermino JM, Broughton Pipkin F, Côté Anne-Marie, et al. Prediction of adverse maternal outcomes in preeclampsia: development and validation of the fullPIERS model. PIERS Study Group. Lancet 2011; 377: 219-27

12. Dahlia Raymond, Erika Peterson. A Critical Review of Early-Onset and Late-Onset Preeclampia. Obstet Gynecol Surv. 2011; 66(8): 497-506.

13. Wójtowicz A, Zembala-Szczerba M, Babczyk D, Kołodziejczyk-Pietruszka M, Lewaczyńska O, Huras H. Early- And Late-Onset Preeclampsia: A Comprehensive Cohort Study of Laboratory and Clinical Findings According to the New ISHHP Criteria. Int J Hypertens. 2019: 4108271.

14. Bartsch E, Medcalf KE, Park AL, Ray JG. Clinical risk factors for preeclampsia determined in early pregnancy: systematic review and meta-analysis of large cohort studies. High Risk of Preeclampsia Identification Group. BMJ 2016; 353: i1753. 
15. Chappell LC, Enye S, Seed P, Briley AL, Poston L, Shennan AH. Adverse perinatal outcomes and risk factors for preeclampsia in women with chronic hypertension: a prospective study. Hypertension 2008; 51: $1002-9$.

16. Duckitt K, Harrington D. Risk factors for preeclampsia at antenatal booking: systematic review of controlled studies. BMJ. 2005 Mar 12; 330(7491): 565

17. ACOG Practice Bulletin No. 132: Antiphospholipid syndrome. Obstet Gynecol. 2012 Dec;120(6):151421.

18. Branch DW, Silver RM, Blackwell JL, Reading JC, Scott JR. Outcome of treated pregnancies in women with antiphospholipid syndrome: an update of the Utah experience. Obstet Gynecol 1992; 80: 614-20.

19. Lima F, Khamashta MA, Buchanan NM, Kerslake S, Hunt BJ, Hughes GR. A study of sixty pregnancies in patients with the antiphospholipid syndrome. Clin Exp Rheumatol 1996; 14: 131-6

20. Yamada H, Atsumi T, Kobashi G, Ota C, Kato EH, Tsuruga N, et al. Antiphospholipid antibodies increase the risk of pregnancy-induced hypertension and adverse pregnancy outcomes. J Reprod Immunol 2009; 79: $188-95$

21. Gris JC, Bouvier S, Nouvellon E, Lissalde-Lavigne G, Mercier E, Balducchi JP, et al. Antiphospholid antibodies and the risk of pregnancy complications. Thromb Res. 2017 Mar; 151 Suppl 1: S34-S37.

22. Alijotas-Reig J, Esteve-Valverde E, Ferrer-Oliveras R, Sáez-Comet L, Lefkou E, Mekinian A, et al. The European Registry on Obstetric Antiphospholipid Syndrome (EUROAPS): A survey of 1000 consecutive cases. Autoimmun Rev. 2019 Apr;18(4): 406-414.

23. Schreiber K, Radin M, Sciascia S. Current insights in obstetric antiphospholipid syndrome. Curr Opin Obstet Gynecol. 2017 Dec;29(6):397-403.

24. Sibai BM. The HELLP syndrome (hemolysis, elevated liver enzymes, and low platelets): much ado about nothing? Am J Obstet Gynecol 1990; 162: 311-6

25. Dollberg S, Haklai Z, Mimouni FB, Gorfein I, Gordon ES. Birth weight standards in the live-born population in Israel. Isr Med Assoc J 2005; 7(5):311-314

26. Report of the National High Blood Pressure Education Program Working Group on High Blood Pressure in Pregnancy. Am J Obstet Gynecol. 2000 Jul; 183(1): S1-S22.

27. Levine JS, Branch DW, Rauch J. The antiphospholipid syndrome. N Engl J Med 2002; 346: 752-63.

28. Branch DW, Andres R, Digre KB, Rote NS, Scott JR. The association of antiphospholipid antibodies with severe preeclampsia. Obstet Gynecol 1989; 73: 541-5.

29. Milliez J, Lelong F, Bayani N, Jannet D, el Medjadji M, Latrous H, et al. The prevalence of autoantibodies during third-trimester pregnancy complicated by hypertension or idiopathic fetal growth retardation. Am J Obstet Gynecol 1991; 165: 51-6.

30. Moodley J, Bhoola V, Duursma J, Pudifin D, Byrne S, Kenoyer DG. The association of antiphospholipid antibodies with severe early-onset preeclampsia. S Afr Med J 1995; 85: 105-7.

31. Miyakis S, Lockshin MD, Atsumi T, Branch DW, Brey RL, Cervera R, et al. International consensus statement on an update of the classification criteria for definite antiphospholipid syndrome (APS). J Thromb Haemost 2006; 4: 295-306

32. Gibbins KJ, Tebo AE, Nielsen SK, Branch DW. Antiphospholipid antibodies in women with severe preeclampsia and placental insufficiency: a case-control study. Lupus. 2018 Oct; 27(12): 1903-1910

33. do Prado AD, Piovesan DM, Staub HL, Horta BL. Association of anticardiolipin antibodies with preeclampsia: A systematic review and meta-analysis. Obstet Gynecol 2010; 116: 1433-1443 
34. Abou-Nassar K, Carrier M, Ramsay T, Rodger MA. The association between antiphospholipid antibodies and placenta mediated complications: A systematic review and meta-analysis. Thromb Res 2011; 128: 77-85

35. Cohen D, Buurma A, Goemaere NN, Girardi G, le Cessie S, Scherjon S, et al. Classical complement activation as a footprint for murine and human antiphospholipid antibody-induced fetal loss. J Pathol 2011; 225: 502-511.

36. Girardi G, Berman J, Redecha P, Spruce L, Thurman JM, Kraus D, et al. Complement C5a receptors and neutrophils mediate fetal injury in the antiphospholipid syndrome. J Clin Invest 2003; 112: 1644-1654

37. Holers VM, Girardi G, Mo L, Guthridge JM, Molina H, Pierangeli SS, et al. Complement C3 activation is required for antiphospholipid antibody-induced fetal loss. J Exp Med 2002; 195: 211-220

38. Salmon JE, Girardi G, Holers VM. Activation of complement mediates antiphospholipid antibody-induced pregnancy loss. Lupus 2003; 12: 535-538

39. Shamonki JM, Salmon JE, Hyjek E, Baergen RN. Excessive complement activation is associated with placental injury in patients with antiphospholipid antibodies. Am J Obstet Gynecol 2007; 196: 167. e1-e5.

40. Erkan D, Derksen WJ, Kaplan V, Sammaritano L, Pierangeli SS, Roubey R, et al. Real world experience with antiphospholipid antibody tests: how stable are results over time? Ann Rheum Dis 2005; 64: 1321-5.

Table 1. Antibody profile of the study population

\begin{tabular}{ll}
\hline Variable & Incidence $(\mathrm{n}=20)$ \\
\hline aCL & 11 \\
B2GP1 & 5 \\
LAC & 10 \\
Double positive & 4 \\
Triple positive & 2 \\
\hline
\end{tabular}

Some participants tested positive for more than one aPL test.

aCL - Anticardiolipin antibody positive; LAC - Lupus anticoagulant positive; B2GP1 - $\beta 2$ Glycoprotein1 antibody positive; Double positive - any 2 of 3 above (aCL, B2GP1, LAC) testing positive in the same individual; Triple positive - all 3 testing positive in the same individual.

Table 2. Demographic and clinical baseline characteristics

\begin{tabular}{llll}
\hline Characteristic & Study cohort - positive aPL $(\mathbf{n = 2 0})$ & Control cohort - negative aPL $(\mathbf{n}=\mathbf{3 5})$ & $\mathbf{P}$ \\
\hline Maternal age (years) & $30.15 \pm 4.64$ & $34.34 \pm 7.02$ & $\mathbf{0 . c}$ \\
Gravidity & $3(1.25-4.75)$ & $2(1-2)$ & $\mathbf{0 . 0}$ \\
Chronic hypertension $_{\text {Diabetes Mellitus }}^{\mathbf{a}}$ & $2(10 \%)$ & $7(20 \%)$ & 0.4 \\
SLE & $1(5 \%)$ & $6(17.1 \%)$ & 0.2 \\
Genetic thrombophilia $^{\mathrm{b}}$ & $2(10 \%)$ & $1(2.9 \%)$ & 0.5 \\
Other $^{\mathrm{c}}$ & $3(15 \%)$ & $4(9.3 \%)$ & 1.0 \\
\hline
\end{tabular}

Data are presented as median (interquartile range) or means (standard deviation) for continuous variables, or as number (percent) for categorical variables.

aPL - Antiphospholipid antibodies; SLE - systemic lupus erythematosus

${ }^{\text {a }}$ Diabetes Mellitus refers to both pre-gestational and gestational diabetes. 
b Genetic thrombophilia - Heterozygous factor V Leiden or heterozygous factor II (prothrombin mutation 20210A)

c Other - includes one case of Behçet's disease and one case of glomerulonephritis

Table 3. Obstetrics outcome and pregnancy complications

\begin{tabular}{llll}
\hline Characteristic & $\begin{array}{l}\text { Study cohort }- \\
\text { positive aPL }(\mathbf{n}=\mathbf{2 0})\end{array}$ & $\begin{array}{l}\text { Control cohort }- \\
\text { negative aPL }(\mathbf{n = 3 5})\end{array}$ & P value \\
\hline $\begin{array}{l}\text { GA at } \\
\text { hospitalization } \\
\text { (weeks) }\end{array}$ & $29(26.28-32)$ & $32(28-33)$ & $\mathbf{0 . 0 5}$ \\
$\begin{array}{l}\text { GA at delivery } \\
\text { (weeks) }\end{array}$ & $30(28.3-32.8)$ & $33(30-34)$ & $\mathbf{0 . 0 2}$ \\
$\begin{array}{l}\text { Birth weight } \\
\text { (grams) }\end{array}$ & $1266.7 \pm 579.6$ & $1567.3 \pm 539.7$ & $\mathbf{0 . 0 5 8}$ \\
$\begin{array}{l}\text { Fetal death } \\
\begin{array}{l}\text { Cesarean section } \\
\text { Length of }\end{array}\end{array}$ & $2(10 \%)$ & 0 & 0.13 \\
hospitalization & $18(90 \%)$ & $31(88.6 \%)$ & 1 \\
post-delivery (days) & $9(6-12)$ & $7(6-9)$ & $\mathbf{0 . 0 3}$ \\
HELLP syndrome & $7(35 \%)$ & & \\
$\begin{array}{l}\text { Eclampsia } \\
\text { Placental abruption }\end{array}$ & $2(10 \%)$ & $9(25.7 \%)$ & 0.67 \\
Pulmonary & $1(5 \%)$ & 0 & 0.13 \\
embolism & 0 & $2(5.7 \%)$ & 1.0 \\
$\begin{array}{l}\text { DIC } \\
\text { Sepsis }\end{array}$ & $1(5 \%)$ & 0 & 1.0 \\
\hline
\end{tabular}

Data are presented as median (interquartile range) or means (standard deviation) for continuous variables, or as number (percent) for categorical variables.

GA - Gestational age; aPL - Antiphospholipid antibodies;HELLP - hemolysis, elevated liver enzymes and low platelet count; DIC - Disseminated intravascular coagulation

Table 4. Neonatal outcome

\begin{tabular}{lll}
\hline Characteristic & Study cohort - positive aPL (n=20) & Control cohort - negative a] \\
\hline Small for gestational age & $5(25 \%)$ & $6(17.1 \%)$ \\
Apgar score [?] 7 at 5-minute & $3(15 \%)$ & $3(8.6 \%)$ \\
Length of NICU hospitalization (days) & $34(16.75-57.75)$ & $30(19.5-76.5)$ \\
Respiratory distress syndrome (RDS) & $10(50 \%)$ & $13(37.1 \%)$ \\
Intra-ventricular hemorrhage (IVH) & $2(10 \%)$ & $5(14.3 \%)$ \\
IVH 3+4 & 0 & $1(2.9 \%)$ \\
periventricular leukomalacia (PVL) & 0 & 0 \\
Necrotizing enterocolitis (NEC) & $1(5 \%)$ & $2(5.7 \%)$ \\
Sepsis & $4(20 \%)$ & $2(5.7 \%)$ \\
Seizures & 0 & $1(2.9 \%)$ \\
Hypoglycemia & $1(5 \%)$ & $4(11.4 \%)$ \\
Hyperbilirubinemia & $12(60 \%)$ & $21(60 \%)$ \\
CPAP or mechanical ventilation & $7(35 \%)$ & $12(34.3 \%)$ \\
Retinopathy of prematurity (ROP) & $6(30 \%)$ & $2(5.7 \%)$
\end{tabular}




\begin{tabular}{lll}
\hline Characteristic & Study cohort - positive aPL (n=20) & Control cohort - negative al \\
\hline Broncho-pulmonary disease (BPD) & $1(5 \%)$ & $3(8.6 \%)$ \\
Perinatal death & $3(15 \%)$ & $1(2.9 \%)$ \\
\hline
\end{tabular}

Data are presented as median (interquartile range) or means (standard deviation) for continuous variables, or as number (percent) for categorical variables.

NICU - Neonatal intensive care unit; CPAP - continuous positive airway press

a Small for gestational age defined as birth weight percentile below the $10^{\text {th }}$ percentile for the gestational age based on customized nomograms. 